\title{
Biomasse végétale et production fourragère sur terres de parcours sous climat méditerranéen ou tropical sec
}

\author{
M Etienne \\ INRA, Unité d'Ecodéveloppement, Site Agroparc, 84914 Avignon Cedex 9, France
}

\begin{abstract}
Summary - After an overview on the regions concerned and a review of the concepts of rangeland, fodder production and pastoral value, the vegetation, productivity and potential improvement of mediterranean and dry tropical rangelands are assessed. The main range vegetation types are described and characterised by their physiognomy, plant morphology and main ecological constraints. Rangeland fodder production is assessed by analysing the main limiting production factors and by comparing the intra and interannual variations of fodder productivity and quality. Vegetation dynamics of some common range types is discussed according to range management techniques (fire, cutting, cultivation) or livestock management criteria (stocking rate, type of animal, fodder system). Some examples of range improvements applied to contrasted ecological and socio-economical conditions are discussed by analysing their impact on soil, fodder species, vegetation dynamics and range management. To conclude, the need of splitting from fodder production models to range management models is evoked.
\end{abstract}

\section{Introduction}

Bien que ce symposium concerne l'ensemble des régions chaudes, notre exposé est limité aux régions méditerranéennes et tropicales sèches car les premières sont les seules sur lesquelles je puisse prétendre à une certaine compétence, et les secondes présentent de fortes similitudes physionomiques voire sont contiguës à des régions méditerranéennes (Sahel et Maghreb, Chili et Argentine, Californie et Basse Californie). De plus, M. Togola de l'IER de Bamako a eu la gentillesse de m'indiquer une abondante bibliographie sur les pâturages du Sahel, bibliographie dont j'ai essayé de tirer le meilleur profit. Tous les pays sur lesquels des données bibliographiques ont été consultées possèdent de vastes parcours utilisés à grande échelle par des troupeaux d'ovins, de caprins ou de bovins.

Mais qu'entendons-nous par parcours? Le concept de parcours est assez large et plastique. II regroupe sous une même appellation des espaces de physionomies variées, pouvant faire l'objet de gestions contrastées sous des conditions climatiques très différentes. Etymologiquement, tout espace parcouru par un troupeau (donc utilisé de manière extensive) est un parcours. Toutefois les terres de parcours sont dans la majorité des cas associées à une végétation naturelle ou spontanée basse. Parfois le terme est même réservé aux pâturages naturels plus ou moins envahis par des arbustes, le terme de prairie naturelle étant réservé au restant de l'espace parcouru. Pour ce qui nous concerne, nous garderons un sens plus général au mot parcours en le définissant comme tout espace naturel non fauché utilisé régulièrement par un troupeau d'animaux domestiques.

Une fois le terme défini, il faut répondre aux deux questions posées par les organisateurs du symposium : quelle productivité pour ces parcours? Quelles possibilités d'améliorations ? Ceci oblige à une ou deux réflexions préalables sur les éléments constitutifs de la productivité d'un parcours et sur la façon d'évaluer cette productivité. En effet, selon le point de vue utilisé, la productivité du parcours pourra être jugée en terme de fonctionnement écologique (richesse floristique, complexité de la structure, adaptation au milieu, résilience,...), de production primaire (phytomasse totale, phytomasse aérienne, phytomasse consommable) ou de production secondaire (valeur nutritive, capacité de charge). Même si l'on se contente d'évaluer le parcours seulement en tant que fournisseur potentiel d'aliment pour des animaux domestiques, la façon d'appréhender la valeur pastorale d'un tel espace pâturé reste très variée. Nous avons essayé de retenir plusieurs indices de valeur 
(phytomasse consommable, valeur pastorale, valeur fourragère, ...) car chacun exprime assez bien une des composantes de la productivité des parcours. Malheureusement, pour une région donnée, il est rare de disposer de l'ensemble de ces indices et donc d'avoir une vision globale sur la productivité des parcours.

\section{La végétation de parcours}

Les parcours des régions méditerranéennes et tropicales sèches sont généralement caractérisés par des végétations pluristratifiées où le tapis herbacé est en concurrence plus ou moins forte avec une strate arbustive plus ou moins dense ou une strate arborée basse et clairsemée. Les espèces ligneuses sont généralement sclérophylles voire épineuses. Elles présentent de fortes similitudes physionomiques et morphologiques d'une région à l'autre, même si elles n'appartiennent pas aux mêmes familles botaniques.

Un premier classement est basé sur les conditions climatiques presque toujours caractérisées par les précipitations moyennes annuelles utilisées comme un indicateur du degré de sécheresse. Ce zonage des grands types de végétation de parcours est parfois amélioré en introduisant soit une mesure plus précise du stress hydrique comme la durée de la période active (Boudet, 1978), soit un facteur limitant complémentaire comme les températures minimales hivernales.

A l'intérieur d'une même zone, la typologie des parcours fait référence au type de substrat dominant et aux strates représentées. Par exemple, on distinguera les maquis du bassin méditerranéen ou le matorral chilien sur sols acides, des garrigues du bassin méditerranéen, de la phrygana grecque ou de la batha israélienne sur sols calcaires. Ces formations sont ensuite découpées en fonction de leurs espèces dominantes en donnant des typologies similaires à celles proposées par Le Houérou (1969) pour la Tunisie ou par Rivas Goday et Rivas Martinez (1963) pour l'Espagne. A l'intérieur de ces grands types, des variantes sont définies en fonction des espèces associées et du couvert végétal. Parallèlement, dans les steppes ou les savanes sahéliennes, le recouvrement relatif arbre/arbuste/herbe permettra de définir différents stades entre la steppe graminéenne et la steppe arborée ou entre la savane herbeuse et la savane boisée (Trochain, 1957). Enfin le degré d'éloignement de la strate herbacée de son état optimum (défini par les conditions de milieu) sous l'effet de la pression anthropique sera utilisé pour positionner un même type de parcours sur une échelle d'appauvrissement (Liacos et Mouloupoulos, 1967), de dégradation voire de désertification (Etienne et al, 1987).

\section{La production des parcours}

Relativement peu de données sont disponibles sur la production globale des parcours, en particulier si l'on tient compte de la biomasse animale non domestique. Et pourtant les parcours sont très souvent associés à des activités de chasse et certaines espèces de la faune sauvage peuvent jouer un rôle de concurrent direct vis à vis des animaux domestiques.

\section{Phytomasse racinaire}

Les quelques travaux concernant la production végétale totale (épigée et hypogée) des parcours montrent que le rapport entre la phytomasse racinaire et la phytomasse aérienne varie de 0,5 à 1,5 . En région méditerranéenne, ce rapport est d'autant plus faible que le parcours est dégradé (Kummerow, 1981; Floret et al, 1983), alors qu'en région tropicale sèche, il augmente quand les conditions hydriques sont plus défavorables (Penning De Vries et Djitèye, 1982 ; Grouzis, 1988).

\section{Phytomasse aérienne}

Un nombre plus important de données est disponible sur la phytomasse épigée, bien que l'apport de la strate arborée dans cette production soit généralement négligé ou estimé de façon très grossière selon le couvert d'arbre dans un secteur écologique donné. Breman et De Ridder (1991) proposent ainsi de rajouter entre 100 et $700 \mathrm{~kg} \mathrm{MS} / \mathrm{ha} / \mathrm{an}$ à la production des steppes arborées du sud du Sahel selon l'unité de paysage concernée. Ovalle et af (1990) estiment à $1 \mathrm{t} \mathrm{MS/ha/an}$ l'apport de feuilles par Acacia caven pour un espinal en condition favorable au Chili. Les données sont encore plus rares en ce qui concerne les fruits, alors que de nombreux 
parcours arborés contiennent des espèces ligneuses produisant des fruits consommables comme les glands de Quercus ou les gousses d'Acacia ou de Prosopis. Des études sur la dehesa espagnole donnent toutefois une fourchette comprise entre 5 et $40 \mathrm{~kg}$ de gland/arbre ce qui représente de 250 à 400 UF/ha/an pour une dehesa moyenne à 44 arbres/ha (Casa de Velazquez, 1987).

Nous avons regroupé dans le tableau I les données concernant plusieurs types de parcours arbustifs adultes classés en fonction du type de climat et des précipitations annuelles moyennes. Nous ne sommes pas allés jusqu'à calculer l'efficacité d'utilisation de la pluie (RUE) car la végétation herbacée des parcours n'est pas exclusivement composée d'annuelles et parce que ce coefficient ne tient pas compte des contraintes édaphiques qui peuvent modifier considérablement les niveaux de production. Ainsi, en Tunisie, une steppe à Lygeum spartum produira $150 \mathrm{~kg} \mathrm{MS} / \mathrm{ha}$ sur dune, $800 \mathrm{~kg}$ sur encroûtement gypseux et
$1300 \mathrm{~kg}$ sur sol alluvial (Floret et al, 1983). De même, en Egypte, un parcours à Echiochilon fruticosum et Helianthemum lippii produira $2 \mathrm{t}$ $\mathrm{MS} / \mathrm{ha}$ en crête, $5 \mathrm{t}$ en dépression non saline et $6 \mathrm{t}$ sur plateau (Abdel-Razik et al, 1988). De plus, à conditions édaphiques égales, la position topographique peut engendrer de fortes différences de production par ses effets sur le bilan d'eau, en particulier en zone sahélienne (Grouzis, 1988). Signalons toutefois que dans les parcours à dominance d'herbacées annuelles la RUE varie en région méditerranéenne de 3,2 à 5,6 en Californie, de 3 à 8,7 en Galilée, de 3,5 à 4,4 au Chili, de 2,5 à 5,3 en Andalousie et de 2,5 à 3,5 en Extrémadure (Joffre, 1987) alors qu'elle va de 1,7 à 8 en zone sahélienne (Grouzis, 1988).

\section{Potentiel pastoral}

Ces valeurs globales ne correspondent évidemment pas au fourrage prélevé par les animaux domestiques même quand des

Tableau I. Phytomasses aériennes de parcours de région chaudes.

\begin{tabular}{|c|c|c|c|c|}
\hline Régions (ou pays) & Parcours à & $\begin{array}{l}\text { Pluviométrie } \\
(\mathrm{mm})\end{array}$ & $\begin{array}{l}\text { Production } \\
\text { (tMS/ha) }\end{array}$ & $\begin{array}{l}\text { Proportion ( } \% \\
\text { consommée }\end{array}$ \\
\hline Naxos (Grèce) & Juniperus phoenicea & 400 & 40,0 & 17 \\
\hline Languedoc (France) & Quercus coccifera & 800 & $13-24$ & \\
\hline Naxos (Grèce) & Phlomis fruticosa & 400 & 8,0 & \\
\hline Macédoine (Grèce) & Quercus coccifera & 620 & $2,0-3,0$ & $30-40$ \\
\hline Arizona (USA) & Larrea tridentata & 440 & 9,0 & 50 \\
\hline Andalousie (Espagne) & Cistus libanotis & 600 & 2,0 & 10 \\
\hline Andalousie (Espagne) & Erica scoparia & 600 & 13,0 & 30 \\
\hline Alexandrie (Egypte) & Echiochilon fruticosum & 300 & $5,0-6,0$ & $15-20$ \\
\hline Matmata (Tunisie) & Rhanterium suaveolens & 200 & 1,0 & 20 \\
\hline Matmata (Tunisie) & Ziziphus lotus & 300 & 1,5 & \\
\hline Matmata (Tunisie) & Arthrophytum scoparium & 200 & 0,5 & \\
\hline Matmata (Tunisie) & Helianthemum lippii & 200 & 0,8 & \\
\hline Arizona (USA) & & 200 & 0,5 & 70 \\
\hline Arizona (USA) & & 350 & 0,7 & 70 \\
\hline Arizona (USA) & & 450 & 1,0 & 70 \\
\hline Crau (France) & Thymus vulgaris & 500 & 1,0 & 90 \\
\hline Corse (France) & Cistus monspeliensis & 700 & 4,5 & 10 \\
\hline Corse (France) & Brachypodium ramosum & 700 & 2,5 & 40 \\
\hline Moyen-Atlas (Maroc) & Juniperus oxycedrus & & 0,2 & 100 \\
\hline Moyen-Atlas (Maroc) & Quercus ilex & & 0,5 & 100 \\
\hline Moyen-Atlas (Maroc) & Pelouse & & 0,8 & 100 \\
\hline Préalpes (France) & Lavandula vera & 900 & 1,0 & 80 \\
\hline Préalpes (France) & Aphyllanthes monspeliensis & 800 & 0,7 & 100 \\
\hline Préalpes (France) & Bromus erectus & 800 & 1,5 & 100 \\
\hline Galilée (Israël) & Pelouse & 600 & 3,3 & 100 \\
\hline Préalpes (France) & Genista villarsii & - & 0,7 & 70 \\
\hline
\end{tabular}


estimations de la phytomasse consommable sont proposées. La transformation de la production fourragère en potentiel de charge animale implique de transformer ces données en appliquant des coefficients tenant compte de la qualité du fourrage et de son taux d'utilisation. Pour le premier critère, deux indicateurs globaux sont généralement utilisés : la valeur pastorale (VP) basée sur la pondération de la composition botanique du parcours par un indice empirique de qualité des espèces herbacées, ou la valeur nutritive du fourrage calculée à partir de tables alimentaires fournissant la quantité d'unités fourragères produites selon l'espèce concernée. Par exemple, les prairies naturelles de Corse ont des VP qui vont de 15 à 60 (Volaire et al, 1990), celles des Préalpes de 10 à 30 (Garde, 1990) alors que celles de la Sierra Norte de Sevilla varient de 5 à 35 (Casa de Velazquez, 1987).

A partir de là, certains auteurs ont cherché à établir des équations leur permettant de prédire le potentiel pastoral du parcours exprimé en UF/ha, unité aisément transformable en charge animale. Pour les parcours des Préalpes, les équations obtenues sont du type UFL = a VP le coefficient «a» variant de 30 à 55 selon le mode d'utilisation du parcours (Senn, 1991) ou bien du type UFL $=a$ VP - $b$ le coefficient " $b$ " variant de 30 à 130 selon la saison d'utilisation (Garde, 1990).

Ces données globales cachent une importante variabilité interannuelle et de fortes variations saisonnières liées à la distribution et à l'intensité des pluies ainsi qu'au régime thermique, en particulier la longueur de la période froide pour les pays du nord de la Méditerranée.

\section{Variations interannuelles}

Les variations interannuelles portent autant sur la production totale que sur la production herbacée, surtout quand celle-ci est à base d'espèces annuelles. Par exemple, Floret et al (1983) montrent, qu'en Tunisie présaharienne, au sein d'un secteur écologique donné, la production du même type de parcours peut varier de 200 à $1500 \mathrm{~kg} \mathrm{MS} / \mathrm{ha}$ selon les années et que parallèlement mais non simultanément, la proportion entre la phytomasse annuelle et la phytomasse pérenne varie de 2 à $85 \%$. Ces variations existent aussi en terme de productivité mais sont d'autant moins marquées que le compartiment arbustif du parcours est bien développé. Ainsi pour une garrigue à Quercus coccifera du sud de la France la productivité oscillera entre 2 et $3,8 \mathrm{t} \mathrm{MS} / \mathrm{ha} / \mathrm{an}$ (Rapp et Lossaint, 1981) alors que dans une steppe à Larrea cuneifolia d'Argentine, elle variera de 2 à $5,5 \mathrm{t} \mathrm{MS} /$ ha/an (Martinez et Dalmasso, 1992).

\section{Variations saisonnières}

Les variations saisonnières portent à la fois sur la distribution de la production au sein de l'année et sur la longueur de la période de production au sein de chaque saison. Dans le cas des parcours arbustifs, les variations sont surtout liées à la phénologie de l'espèce arbustive dominante. Par exemple, un parcours à Eremophila gilesii d'Australie voit sa production varier de 1,5 $\mathrm{t} \mathrm{MS/ha}$ en fin d'hiver à 2,5 $t$ en automne (Burrows, 1972). Parallèlement, dans une phrygana de Crète, la variation saisonnière de la production herbacée, très importante selon l'altitude, est presque totalement gommée par la variation saisonnière de la production arbustive (Kyriakakis et Papanastasis, 1993). Dans le cas des parcours herbacés du Sahel, les variations sont liées à la durée de la période sans déficit hydrique. Par contre, dans certaines régions méditerranéennes, les variations des températures minimales hivernales peuvent modifier profondément la répartition saisonnière des courbes de production. Ainsi, en Sardaigne, $70 \%$ de la production fourragère annuelle sera obtenue en hiver une année vs $30 \%$ l'année suivante (Sulas et al, 1993).

\section{Qualité}

L'ensemble des facteurs mentionnés joue également sur la qualité du fourrage produit par le parcours. Cette notion de qualité est toutefois plus délicate à saisir dans le cas des parcours arbustifs ou boisés. En effet, autant la qualité d'un fourrage herbacé peut être correctement estimée en combinant les espèces dominantes, le stade phénologique et le niveau de fertilisation, autant la qualité d'un fourrage provenant de ligneux est plus difficile à appréhender. Beaucoup de travaux estiment cette valeur nutritive sur la base d'analyses chimiques plus ou moins complexes de la 
digestibilité et de la teneur en protéine. Certains tempèrent ces valeurs par la teneur en phytoconstituants secondaires des ligneux (Nastis et Papanastasis, 1993). D'autres essaient de classer les espèces de parcours en fonction de leur appétibilité mesurée à travers soit le comportement des animaux, soit le taux d'abroutissement. Le cas des fruits est encore plus complexe quand on voit que les glands produits par les dehesas espagnoles dont la valeur nutritive équivaut à $0,6 \mathrm{UF} / \mathrm{kg}$ ont une valeur de transformation en viande de porc très différente selon l'espèce de chêne concerné. Selon Ruperez (1957), il faut en effet 9,12 ou $14 \mathrm{~kg}$ de gland pour produire 1 $\mathrm{kg}$ de viande de porc selon que la glandée provient respectivement de Quercus rotundifolia, $Q$. faginea ou $Q$. suber.

\section{Effet de l'âge}

Etant donné la difficulté de datation précise des peuplements arbustifs adultes, peu d'études sur parcours mentionnent leur âge. Or, l'allocation des ressources entre racines, bois et feuilles se fait de façon différente aux différents stades de la vie d'un parcours entre deux modifications ou perturbations majeures (incendie, mise en culture, succession naturelle). Des travaux récents fournissent des données sur l'évolution du rapport phytomasse herbacée/phytomasse arbustive (Etienne et al, communication personelle) ou sur la proportion feuille/tige sur parcours arbustifs (Merino et al, 1990 ; Armand et al, 1993). Ils ouvrent la voie à l'élaboration de modèles prédictifs de la productivité et de la production des parcours similaires aux modèles élaborés par les écologues sur les formations arbustives non pâturées comme le modèle de Chew et Chew (1965) sur la productivité des peuplements de Larrea tridentata en Californie.

\section{Gestion pastorale}

Enfin, les modalités de gestion pastorale influent beaucoup sur l'état d'équilibre entre les strates composants le parcours et jouent donc un rôle déterminant sur leur dynamique. Le chargement et la saison d'utilisation sont les deux principaux facteurs qui modifient substantiellement la production du parcours et la part de fourrage consommable dans cette production. Par exemple, une steppe du
Colorado dominée par Bouteloua gracilis et Buchloe dactyloides, produit en moyenne 0,75 t MS/ha/an pour une précipitation annuelle moyenne de $320 \mathrm{~mm}$. Dans les secteurs pâturés depuis plus de 50 ans, cette productivité se maintient entre 0,71 et 0,68 avec un chargement respectivement faible et moyen, mais chute à 0,57 avec un chargement fort (Milchunas et al, 1994). D'autres éléments comme la distance à certains équipements attractifs créés pour faciliter la gestion des animaux (bergerie, points d'eaux,...) peuvent jouer sur la productivité des parcours. OuledBelgacem et Papanastasis (1995) ont même proposé une équation permettant de corriger la production d'un parcours en Crète en fonction de la distance à la bergerie ou au point d'eau principal.

\section{Possibilités d'amélioration}

Les parcours sous climat méditerranéen ou tropical sec présentent souvent des signes de dégradation liés soit à des pratiques de gestion inconsidérées, soit à un déséquilibre entre chargement animal et potentiel pastoral. Le feu pastoral en Corse (Joffre, 1982) ou la mise en culture trop fréquente des dehesas d'Espagne (Pérez-Férnandez et GomezGutiérrez, 1995) sont deux bons exemples de gestion inconsidérée. Le deuxième processus peut se traduire soit par une surutilisation des ressources pastorales avec les phénomènes de surpâturage que l'on rencontre fréquemment dans les pays du Maghreb ou du Sahel, soit par une sous-utilisation qui entraîne un embroussaillement progressif et une recrudescence des incendies comme en région méditerranéenne française (Etienne et al, 1994) ou en Espagne (Moreno et al, 1994). La remise en marche de ces parcours passe par la mise au point et le développement de techniques d'améliorations pastorales adaptées aux différentes conditions écologiques et socio-économiques. Ces techniques interviennent soit sur la structure ou le niveau trophique du sol, soit sur la composition botanique du parcours, soit sur les modalités de gestion pastorale. Nous avons regroupé sur le tableau II quelques données comparant la production fourragère de plusieurs types de parcours selon différentes techniques d'améliorations. 
Tableau II . Evolution de la production fourragère sur parcours ou prairie naturelle améliorés exprimées en $\mathrm{kg}$ de matière sèche par hectare.

\begin{tabular}{|c|c|c|c|c|c|c|c|c|c|}
\hline \multirow{2}{*}{ arbuste dominant } & \multirow{2}{*}{ techniques } & \multirow{2}{*}{$\begin{array}{l}\text { témoin } \\
\text { herb. }\end{array}$} & \multicolumn{2}{|c|}{ année 1} & \multicolumn{2}{|c|}{ année 3} & \multirow{2}{*}{\multicolumn{2}{|c|}{$\begin{array}{l}\text { année } 5 \\
\text { herb. lign. bas }\end{array}$}} & \multirow{2}{*}{ Ref. } \\
\hline & & & herb. & lign. bas & herb. & lign. bas & & & \\
\hline Quercus coccifera & feu + brome & & 500 & 800 & 1100 & 6400 & & & (1) \\
\hline Phlomis fruticosa & coupe + fert. & 2600 & 6400 & 300 & & & & & (2) \\
\hline Phlomis fruticosa & brul. + fert. & 2600 & 5600 & 500 & & & & & (2) \\
\hline Sarcopotherium sp. & fert. $(P)$ & 500 & 2800 & & 4100 & & 3000 & & (3) \\
\hline Sarcopotherium sp. & $\begin{array}{l}\text { phytocide } \\
+ \text { fert. }(P)\end{array}$ & 900 & 2200 & & 3000 & & 2800 & & (3) \\
\hline Cistus monsp. & $\begin{array}{l}\text { coupe + }(P) \\
+ \text { Trèfle }\end{array}$ & 100 & 1300 & 200 & 1700 & 1200 & 600 & 2200 & (4) \\
\hline Cistus monsp. & coupe $+(\mathrm{N})$ & 500 & 1500 & 100 & 2800 & 800 & 2500 & 1400 & (4) \\
\hline Erica arborea & $\begin{array}{l}\text { coupe + }(N) \\
+ \text { Dactyle }\end{array}$ & 100 & 1400 & 100 & 3300 & 700 & 2700 & 4500 & (4) \\
\hline Erica arborea & coupe $+(\mathrm{N})$ & 300 & 1000 & 100 & 3800 & 400 & 800 & 3700 & (4) \\
\hline
\end{tabular}

(herb. : herbacées ; lign. bas : ligneux bas ; brul. : brûlage ; fert. : fertilisation ; $\mathrm{P}$ : phosphore ; $\mathrm{N}$ : azote.)

\section{PRAIRIES NATURELLES}

\begin{tabular}{|c|c|c|c|c|c|c|}
\hline Site & techniques & témoin & traitement- & aitement & aitement-3 & Ref. \\
\hline Alentejo (Portugal) & $T /-/ P / S$ & 3500 & & 5000 & 6200 & (5) \\
\hline Toscane (Italie) & $\mathrm{T} / \mathrm{N} / \mathrm{P} / \mathrm{N}+\mathrm{P}$ & 1300 & 2200 & 1800 & 2600 & (6) \\
\hline Minho (Portugal) & $\mathrm{T}$ / Ca faible / Ca fort & t 1200 & 2700 & 3700 & & (7) \\
\hline Apulie (Italie) & $T / N /-/ S$ & 4300 & 5600 & & 5700 & (8) \\
\hline Extremadura (Espagne) & $T /-/ P$ & 1000 & & 1100 & & (9) \\
\hline Extremadura (Espagne) & $\mathrm{T} /$ fumier & 1100 & 3000 & & & (10) \\
\hline Extremadura (Espagne) & $T /$ fumier & 1100 & 3300 & & & (10) \\
\hline Extremadura (Espagne) & $T /$ fumier & 1800 & 3800 & & & (10) \\
\hline Extremadura (Espagne) & T/fumier & 1000 & 2100 & & & (10) \\
\hline Pouilles (Italie) & $T / N / P / N+P$ & 3200 & 3000 & 3900 & 5100 & (11) \\
\hline Pouilles (Italie) & $T / N / P / N+P$ & 1600 & 3400 & 4400 & 3800 & (11) \\
\hline Pouilles (Italie) & $\mathrm{T} / \mathrm{N} / \mathrm{P} / \mathrm{N}+\mathrm{P}$ & 3600 & 3700 & 4900 & 4200 & (11) \\
\hline Pouilles (Italie) & $T / N / P / N+P$ & 2300 & 4000 & 4100 & 4100 & (11) \\
\hline
\end{tabular}

( $\mathrm{T}$ : témoin ; $\mathrm{P}$ : phosphore; $\mathrm{N}$ : azote ; $\mathrm{S}$ : soufre ; $\mathrm{Ca}:$ calcium).

Références bibliographiques (Ref.)
(1) Papanastasis et Platis, 1990
(7) Moreira et al, 1993
(2) Kandrelis et Papanastasis, 1993
(8) Corleto, 1993
(3) Henkin et al, 1995
(4) Etienne et al, résultats non publiés
(9) Moreno et al, 1993
(5) Fragoso De Almeida et Fernandes, 1990
(10) Olea et al, 1987
(6) Battistelli et al, 1990
(11) Martiniello et al, 1995 


\section{Amélioration du sol}

Les techniques les plus couramment utilisées cherchent à augmenter la capacité de réserve en eau du sol et à relever sa fertilité. Au Sahel, le grattage de la croûte supérieure du sol effectué à la bonne saison peut permettre la réinstallation d'un tapis graminéen sur parcours dégradé (Toutain, 1977). Mais tout travail du sol (grattage, poquets, labour) gagne à être accompagné d'une fertilisation au moins phosphatée pour favoriser l'installation d'espèces de meilleure qualité (Cissé, 1982). La fertilisation n'y a jamais été testée à grande échelle, même si quelques rares essais expérimentaux montrent qu'une fertilisation phosphatée multiplie par 1,5 à 1,8 la production et par 3 la teneur en phosphore du fourrage (Breman et Krul, 1982).

En région méditerranéenne, la fertilisation directe sur prairie naturelle ou après débroussaillement sur parcours arbustif est couramment préconisée. Elle donne des résultats spectaculaires sur prairies naturelles ou dans les parcours où le potentiel en espèces fourragères spontanées est élevé, en particulier ceux riches en légumineuses. Par exemple, en Extrémadure une légère fertilisation phosphatée suffit à faire passer la production fourragère des parcours de dehesa de 900 à $1500 \mathrm{~kg} \mathrm{MS/ha} \mathrm{(Olea} \mathrm{et} \mathrm{al,} \mathrm{1987).} \mathrm{De}$ même, en Corse, le développement du trèfle souterrain permet de faire passer la productivité d'un parcours à Cistus monspeliensis de 0,3 à $3 \mathrm{t}$ MS/ha/an (Etienne, 1977). Quand la strate herbacée du parcours est moins riche, la fertilisation ne permet de multiplier la production que par 2 ou 3 . Ainsi, des parcours à Brachypodium retusum du sud de la France passent de $1 \mathrm{t}$ à $3 \mathrm{t} \mathrm{MS/ha/an} \mathrm{à} \mathrm{la}$ suite d'une fertilisation azotée régulière (Poissonet et al, 1981 ; Joffre et Casanova, 1983).

La fertilisation a également un effet intéressant en stimulant la production à certaines saisons de l'année, ce qui peut permettre de combler un déficit fourrager et d'économiser du complément ou des déplacements. La fertilisation azotée en fin d'hiver permet ainsi, dans le nord du bassin méditerranéen, d'anticiper la pousse de printemps (Battistelli et al, 1990 ; Coppel et Etienne, 1992). Par contre, une fertilisation azotée d'automne sera sans effet sur la production hivernale d'un parcours
d'Extrémadure alors qu'un apport de phosphate à la même saison multipliera par deux cette production (INIA, 1984).

\section{Amélioration de la composition botanique}

L'introduction d'espèces fourragères sur parcours concerne aussi bien le compartiment ligneux que la strate herbacée. De nombreux travaux ont été réalisés en région méditerranéenne sur cet aspect et le lecteur pourra en avoir une vision détaillée en consultant les derniers compte-rendu des réunions du groupe de travail de la FAO sur les pâturages méditerranéens (Mansat, 1987 ; Corleto, 1990 ; Papanastasis, 1993 ; Etienne, 1995).

L'introduction d'espèces herbacées sur parcours se fait soit par sursemis (apport de graines sans travail du sol), soit par semis (déssouchage + travail du sol + apport de graines) et s'accompagne dans la majorité des cas d'une fertilisation, au moins l'année d'installation. Des résultats spectaculaires ont été enregistrés en région méditerranéenne, en particulier sur sols acides. Les semis de Trifolium subterraneum ont permis de multiplier par 10 la production fourragère des "montados» de l'Alentejo au Portugal alors que les sursemis de la même espèce dans le sud de la France ont fait passer la production fourragère des maquis de 0,5 à $4 \mathrm{t} \mathrm{MS} / \mathrm{ha} / \mathrm{an}$ (Etienne, 1991; Masson et al, 1991). Toutefois la maîtrise de l'embroussaillement sur ce type de parcours pose problème et il faut pratiquer périodiquement des débroussaillements d'entretien pour maintenir le parcours à son meilleur niveau de productivité. En effet, les repousses arbustives, même si elles sont partiellement consommables, créent une concurrence qui au bout de 3-4 ans a un effet dépressif sur la production fourragère herbacée.

L'introduction d'arbres ou arbustes fourragers permet de créer une ressource d'appoint en période estivale en jouant sur le déphasage phénologique de la pousse des feuilles d'arbres ou d'arbustes. De bons résultats ont été obtenus avec les plantations d'Atriplex dans les zones arides méditerranéennes avec une productivité de 1,5 à 2 t MS/ha/an en Afrique (Le Houérou, 1992) et de 2 à 2,5 t au Chili (Olivares et Gasto, 1981). Dans les pays du nord de la Méditerranée, les rendements obtenus varient 
de 300 à $500 \mathrm{~g} \mathrm{MS} /$ plante pour des espèces comme Morus alba, Robinia pseudoacacia et Amorpha fruticosa (Papanastasis, 1995).

\section{Amélioration de la gestion pastorale}

Les techniques précédemment mentionnées présentent toutes un coût d'investissement à l'hectare de parcours élevé et des coûts d'entretien plus faibles mais réguliers au moins sur le moyen terme. Une autre alternative d'amélioration consiste donc à mieux ajuster la gestion pastorale à la production et au mode de régénération du parcours.

Une première possibilité consiste à trouver le mode de conduite du troupeau permettant une meilleure utilisation possible du parcours à long terme. Par exemple, la comparaison de quatre types de gestion pastorale combinant chargement et période d"utilisation au Texas montrent des différences de production de plus de $500 \mathrm{~kg} \mathrm{MS} / \mathrm{ha} / \mathrm{an}$ entre un pâturage continu à forte charge et un pâturage continu modéré (Kothmann et al, 1978).

Une deuxième possibilité consiste à rechercher une utilisation maximale de la ressource fourragère du parcours, en particulier une bonne consommation du fourrage provenant des ligneux. Plusieurs éléments de conduite peuvent être utilisés pour obtenir ce résultat (Etienne et al, 1995) : augmenter la pression de pâturage (charge instantanée), augmenter la fréquence d'utilisation du parcours (varier les saisons), assurer une ration azotée minimale directe (soupade sur légumineuses) ou indirecte (complément type paille ammoniaquée ou mélasse-urée), utiliser des races rustiques habituées au parcours.

Enfin, à l'opposé, une troisième possibilité consiste à favoriser la récupération d'espèces fourragères sur parcours dégradés. Les solutions proposées consistent généralement à intégrer une période de mise en défens ou de mise en repos, à une saison opportune pour la régénération et pendant un temps suffisamment long pour permettre une reconstitution du tapis herbacé. Ces solutions généralement très efficaces sur le plan technique (Kothmann et al, 1978 ; Grouzis, 1988 ; Tsiouvaras et al, 1993) posent de gros problèmes de mise en oeuvre car elles trouvent difficilement leur place dans les systèmes fourragers traditionnels.

\section{Conclusion}

La production fourragère d'un parcours des régions chaudes est donc quelque chose de très complexe à évaluer car elle concerne souvent plusieurs strates de végétation ou plusieurs types de végétaux, elle présente de fortes variations saisonnières et interannuelles et elle est très liée au mode d'utilisation. C'est sur ce dernier point que je voudrais étayer ma conclusion, en discutant rapidement de la modélisation de la production fourragère des parcours. De nombreux modèles sont basés sur les courbes de croissance de la strate herbacée en fonction de différents paramètres climatiques: sommes de température (George et al, 1989), sommes d'ETR (Coppel et Etienne, 1992) parfois combinés avec la texture et la fertilité du sol (Qarro et De Montard, 1992 ; Bergez et Msika, 1995). Très peu de modèles essayent d'intégrer les variations saisonnières ou de prendre en compte l'effet de la gestion sur les courbes de croissance. Les modèles actuels restent donc des modèles de production optimale en fonction d'une gestion donnée. Mais si l'on étudie un peu les systèmes fourragers, on se rend vite compte que les parcours y tiennent une place différente selon le système d'élevage ou les autres ressources fourragères disponibles sur l'exploitation (Papanastasis, 1990; Etienne et al, 1990). Il est alors nécessaire de réfléchir à une deuxième catégorie de modèles qui évaluent la productivité d'un parcours en fonction de sa période et de son intensité d'utilisation et donc en concordance avec les besoins du troupeau comme ceux proposés par De Ridder et Breman (1993) pour le Sahel. Ce n'est qu'en créant des ponts entre ces deux types de modèles que l'on pourra vraiment prétendre connaître la productivité des parcours sous climat méditerranéen ou tropical sec.

\section{Références}

Abdel-Razik M, Ayyad M, Heneidy S (1988) Phytomass and mineral composition in range biomass of a Mediterranean arid ecosystem (Egypt). Oecol Plant 9, 359-370

Armand D, Etienne M, Legrand C, Maréchal J, Valette JC (1993) Phytovolume, phytomasse et relations structurales chez quelques arbustes méditerranéens. An Sci Forest 50, 79-89 
Battistelli A, Olimpieri G, Cannata F, Brugnoli E (1990) Effect of fertilization on Mediterranean pasture production in the "Maremma Laziale" region. In: Proc 6th Meet FAO Eur Sub-Network on Mediterr Past Fodder Crops 91-94

Bergez JE, Msika B (1995) A sylvopastoral model for the EU. Cah Opt Mediterr 12, 231-234

Boudet G (1978) Manuel sur les pâturages tropicaux et les cultures fourragères. IEMVT, Paris, $258 p$

Breman H, De Ridder N (1991) Manuel sur les pâturages des pays sahéliens. Karthala, Paris, $485 p$

Breman H, Krul JM (1982) La stimulation des Légumineuses. In: La productivité des pâturages sahéliens ( $F$ Penning De Vries, M Djitèye, eds) Pudoc, Wageningen, 434-439

Burrows W (1972) Productivity of an arid zone shrub community in south-western Queensland. Austr $J$ Botany 20, 317-329

Casa de Velazquez (1987) Supervivencia de la sierra Norte de Sevilla. Ministère de l'Agriculture, Madrid, $376 \mathrm{p}$

Chew R, Chew A (1965) The primary productivity of a desert-shrub (Larrea divaricata) community. Ecolog Monogr 35, 355-375

Cissé I (1982) La régénération des terrains dégradés. In: La productivité des pâturages sahéliens ( $F$ Penning De Vries, M Djitèye, eds) Pudoc, Wageningen, 440-449

Coppel B, Etienne M (1992) Un modèle de production prairiale dans les Préalpes: les pelouses à Bromus erectus. Fourrages 131, 271-282

Corleto A (1990) Development and preservation of low input mediterranean pastures and fodder systems. Univ Bari, $218 \mathrm{p}$

Corleto A (1993) Influence of cutting frequencies, nitrogen fertilization and reseeding on DMY of natural pastures in Apulia region. FAO REUR technical series $28,74-79$

De Ridder N, Breman H (1993) A new approach to evaluating rangeland productivity in sahelian countries. In: Range ecology at disequilibrium ( $R$ Behnke, I Scoones, C Kerven, eds) ODI Londres, 104-117

Etienne M (1977) Un essai d'amélioration des ressources pastorales de la végétation en Corse. Fourrages 71, 83-92

Etienne M (1991) Sursemis sur parcours débroussaillés dans le Sud Est de la France. Fourrages 127, 321-324

Etienne M (1995) Systèmes sylvopastoraux pour un environnement, une agriculture et une économie durables. In: Cah Opt Mediterr 12, FAO/CIHEAM/INRA, $280 p$

Etienne M, Derzko M, Rigolot E (1995) Impact du pâturage sur les arbustes dans des aménagements sylvopastoraux à objectif de prévention des incendies. Cah Opt Mediterr 12, 217-220

Etienne M, Faúndez L, Valdes J (1987) La desertizacion en la zona árida mediterránea de Chile. Terra Arida 6, 1-93

Etienne M, Hubert B, Lachaux M, Couix N, Jullian P, Lasseur J, Lecrivain $E$, Legrand $C$, Leouffre MC, Napoleone M (1990) Territoire pastoral et espace forestier: essai d'analyse des pratiques de pâturage. In: Modélisation systémique et système agraire. Décision et organisation (Brossier, Vissac, Le Moigne, eds), INRA Paris, 255-267

Etienne M, Mas I, Rigolot E (1994) Combining techniques of fuel reduction for fuel-break maintenance in the French mediterranean region. In: Proc 2nd int Conf Forest Fire Res, Coimbra, Portugal, 713-721

Floret C, Le Floc'h E, Pontanier R (1983) Phytomasse et production végétale en Tunisie présaharienne. Oecol Plant 4, 133-152

Fragoso de Almeida JP. Fernandes AJG (1990) Improvement of Mediterranean annual type pastures, under covering of olive grove, in Castelo Branco (Portugal). In: Proc 6th meet FAO Eur Sub-Network on Mediterranean Pastures and Fodder, Crops 69-72

Garde L (1990) Ressources pastorales en Haute Provence et modelisation de la relation végétation-troupeau. Thèse Ecologie, Univ AixMarseille III, $172 p$

George M, Williams W, Mc Dougald N, Clawson J, Murphy A (1989) Predicting peak standing crop on annual range using weather variables. J Range Manag 42, 508-513

Grouzis M (1988) Structure, productivité et dynamique des systemes écologiques sahéliens. ORSTOM, Paris, $335 \mathrm{p}$

Henkin Z, Noy-Meir I, Gutman M, Seligman N (1995) The effect of phosphorus nutrition, shrub control and fire on the dynamics of Mediterranean batha vegetation in Galilee, Israel. Cah Opt Mediterr $12,125-128$

INIA (1984) Mejora de pastos en secanos semiaridos de suelos acidos. Ministère Agriculture, Madrid, $218 \mathrm{p}$

Joffre R (1982) Réflexions sur le feu pastoral en Corse. Premiers résultats de l'étude comparative des parcours incendiés et des parcours améliorés dans le centre de la Corse. Fourrages 91, 73-98

Joffre R, Casanova JB (1983) Le développement des ressources fourragères des parcours en Corse de l'intérieur. Fourrages 93, 51-84

Joffre R (1987) Contraintes du milieu et réponses de la végétation herbacée dans les dehesas de la Sierra Norte. Thèse Ecologie, USTL Montpellier, $186 \mathrm{p}$ 
Kandrelis S, Papanastasis V (1993) Improvement of phryganic rangelands in Epirus, western Greece. FAO REUR technical series 28, 159-162

Kothmann M, Waldrip W, Mathis G (1978) Rangeland vegetation of the Texas Rolling Plains: response to grazing management and weather. In: Proc /st inter Range Cong Denver, 606-609

Kummerow J (1981) Structure of roots and root systems. In: Mediterranean-type shrublands (Di Castri, Goodall, Specht, eds), 269-288

Kyriakakis A, Papanastasis V (1993) Seasonal changes of rangeland production in relation to altitude in western Crete. FAO REUR technical series $28,155-158$

Le Houérou HN (1969) La végétation de la Tunisie steppique. Ann Inst Nat Rech Agron Tunisie 42 (5), 1-624

Le Houérou HN (1992) The role of saltbushes (Atriplex spp.) in arid land rehabilitation in the Mediterranean Basin: a review. Agrof Syst 18, 107-148

Liacos L, Mouloupoulos C (1967) Contribution to the identification of some range types of Quercus coccifera. Gr For Res Bull 16, 1-54

Mansat P (1987) Ressources fourragères et pastorales intégrées dans les systèmes de production en milieu méditerranéen. FAO, $160 \mathrm{p}$

Martinez EM, Dalmasso AD (1992) Litter yield in srhubs of larrea in the andean piedmont of Mendoza, Argentine. Vegetatio 101(1), 21-33

Martiniello P, D'Agnano G, Padalino O, Nardelli F (1995) Effect of fertilization on flora, biomass and seed production and soil fertility in 4 natural pastures of the Mediterranean basin. Cah Opt Mediterr 12, 87-90

Masson Ph, Goby JP, Rochon JJ, Anthelme B (1991) Place des améliorations pastorales à base de trèfle souterrain dans les systèmes d'élevage liés à la prévention des incendies en zone méditerranéenne acide (France). Proc 4th International Rangeland Congress, Montpellier 2, 794-796

Merino O, Martin M, Martin A, Merino J (1990) Successional and temporal changes in primary productivity in two mediterranean schrub ecosystems. Acta Oecol 11, 103-112

Milchunas D, Forwood J, Lauenroth W (1994) Productivity of long-term grazing treatments in response to seasonal precipitation? J Range Management 47(2), 133-139

Moreira N, Coutinho J, Trindade H (1993) Establishment of rainfed Mediterranean annualtype pastures. The efficiency of liming on acid soils. FAO REUR technical series $28,66-69$

Moreno JM, Vásquez A, Pérez B, Faraco AM (1994) Fire and land-use change interactions in Central Spain. Proc MEDECOS VII Via del Mar, Chili, 41-45
Moreno V, Bueno C, Santos A (1993) Natural pasture fertilization with superphosphate in the dehesa ecosystem. FAO REUR technical series 28 , 80-84

Olea L, Paredes J, Verdasco P (1987) Mejora de los pastos de la dehesa. Min Agric, Madrid, 87-110

Olivares A, Gasto J (1981) Atriplex repanda: organizacion y manejo de ecosistemas con arbustos forrajeros. Univ du Chili, Santiago, $300 \mathrm{p}$

Ouled Belgacem A, Papanastasis V (1995) Impact of grazing practices on rangeland vegetation of western Crete. Cah Opt Mediterr 12, 209-212

Ovalle C, Aronson J, Del Pozo A, Avendano J (1990) The Espinal, agroforestry systems of the Mediterranean-Type climate region of Chile. Agroforestry Systems 10, 213-239

Papanastasis V (1990) Pastoral and forage systems in Greece. In: Proc 6th meet FAO Eur SubNetwork on Mediterranean Pastures and Fodder Crops, 177-180

Papanastasis $V$ (1993) Management of mediterranean shrublands and related forage resources. REUR Technical Series, FAO, 28 , $221 \mathrm{p}$

Papanastasis V (1995) Selection and utilization of cultivated fodder trees and shrubs. In: Mediterranean extensive livestock production systems. CIHEAM Zaragoza, $119 \mathrm{p}$

Papanastasis V, Platis $P$ (1990) Effects of range grass seeding on a wildburned Kermes Oak shrubland in Greece. Proc 6th Meet FAO Eur Sub-Network on Mediterranean Pastures Fodder Crops, 25-28

Penning De Vries F, Djitèye M (1982) (eds) La productivité des pâturages sahéliens. Pudoc, Wageningen, $525 \mathrm{p}$

Pérez-Férnandez MA, Gomez-Gutiérrez JM (1995) Evolution of the tree cover (Quercus pyrenaica and Quercus ilex) in a dehesa over the last 100 years. Cah Opt Mediterr, 12, 259-262

Poissonet J, Poissonet P, Thiault M (1981) Development of flora, vegetation and grazing value in experimental plots of a Quercus coccifera garrigue. Vegetatio 46, 93-104

Qarro M, De Montard FX (1992) Etude de la productivité des parcours de la zone d'Ain-Leuh, 2- Modélisation de la production d'herbe : interactions climats $\mathrm{x}$ sol $\mathrm{x}$ recouvrement arboré. Agronomie 12, 489-501

Rapp M, Lossaint $P$ (1981) Some aspects of mineral cycling in the garrigue of Southern France. In: Mediterranean-type shrublands (Di Castri, Goodall, Specht, eds), 269-288

Rivaz-Goday S, Rivas-Martinez S (1963) Esutio y clasificacion de los pastizales espanoles. Ministetio de Agricultura, Madrid, $270 p$ 
Ruperez A (1957) La encina y su tratamientos. Selvicolas, Madrid, 153p

Senn O (1991) Impact du pâturage ovin sur la végétation de parcours clôturés et fertilisés dans les Préalpes du Sud. Thèse Ecologie, Univ AixMarseille III, $155 \mathrm{p}$

Sulas L, Porqueddu C, Carreda S, Bullitta P (1993) Use of annual legumes for pasture improvement in Mediterranean environment. FAO REUR technical series $28,32-35$

Toutain B (1977) Essais de régénération mécanique de quelques parcours sahéliens dégradés. Rev Elev Med Vet Pays Trop 29, 191-198
Trochain JL (1957) Accord interafricain sur les types de végétation de l'Afrique tropicale. Bull inst Etud Centrafr 13-14, 55-93

Tsiouvaras C, Koukoura Z, Ainalis A, Platis P (1993) Dynamic relationship between long-term sheep grazing and range productivity in a semi-arid grassland. FAO REUR technical series 28 . 151-154

Volaire F, Godron M, Lelièvre F (1990) Les formations herbacées de Corse. 2. Essai de validation de la typologie par la caractérisation de la structure végétale des types. Agronomie 10, 233-241 